\title{
Lesiones intraorales de glándulas salivales menores*
}

\author{
Drs. MARÍA ANGÉLICA MATAMALA S. ${ }^{1}$, MIGUEL GONZÁLEZ P. ${ }^{2}$, \\ GONZALO ROSSEL D. ${ }^{2}$, Int. CONSTANZA NIEME S. ${ }^{3}$ \\ 1 Servicio de Cirugía, Hospital Clínico San Pablo, Coquimbo. \\ 2 Servicio de Cirugía Hospital Barros Luco-Trudeau, Santiago. \\ 3 Interna de Medicina, Facultad de Medicina Universidad Católica del Norte, Coquimbo. \\ Chile.
}

\begin{abstract}
Intraoral lesions of minor salivary glands. Analysis of 59 patients

Background: Most salivary gland lesions are benign. Tumors are uncommon. Pleomorphic adenoma and cystic adenoid carcinoma are the most common benign and malignant lesions. Aim: To assess the incidence management and evolution of intraoral lesions among patients consulting in a head and neck surgical service. Material and Methods: Retrospective review of medical records of patients consulting for intraoral lesions of minor salivary glands between 1975 and 2009 in a head and neck surgical service. Results: Thirty five females and 24 males are reported. Forty eight (81\%) had benign lesions. Of these, 35 (73\%). were non neoplastic lesions. Eleven patients had malignant lesions. Of these, four (36\%) had a mucoepidermoid carcinoma, two (18\%) had an adenoid-cystic carcinoma, two (18\%) had an acinous cell carcinoma and one patient each had an undifferentiated carcinoma, an adenocarcinoma and an epidermoid carcinoma. Conclusions: In this series of patients, benign lesions predominated. Benign lesions were excised and the management of malignant lesions depended on the type of tumor.
\end{abstract}

Key words: Salivary glands, tumors, intraoral lesions.

Resumen

Introducción: Las lesiones de glándulas salivales menores son en su mayoría benignas. Los tumores son infrecuentes y conforman un grupo heterogéneo, siendo el adenoma pleomorfo la neoplasia benigna más frecuente y el carcinoma adenoideo-quístico el tumor maligno más encontrado. Objetivos: El propósito de este estudio es evaluar la incidencia, manejo y evolución de las lesiones intraorales de glándulas salivales menores en el Servicio de Cirugía de Cabeza y Cuello del Hospital Barros Luco-Trudeau. Material y Método: Se presenta estudio retrospectivo de 59 casos tratados entre 1975 y 2009 en dicho hospital. Resultados: Encontramos 35 mujeres y 24 hombres. En 48 pacientes $(81,3 \%)$ se encontró lesiones benignas, de las cuales 35

* Recibido el 24 de octubre de 2011 y aceptado para publicación el 5 de marzo de 2012.

Los autores no declaran conflictos de interés.

Correspondencia: Constanza Nieme S.

Río Itata \#4193, Rosario de Peñuelas, Coquimbo, Chile. Código Postal: 1801379.

constanza.n.s@gmail.com 
(73\%) correspondieron a lesiones no neoplásicas y $13(27 \%)$ a neoplasias. Las neoplasias malignas fueron 11 (18,6\% del total), 4 (36,3\%) con Ca mucoepidermoide, 2 (18\%) con Ca adenoideo-quístico, 2 (18\%) con Ca de células acinosas, 1 (9\%) con Ca indiferenciado, 1 (9\%) con adenocarcinoma y 1 (9\%) con Ca epidermoide. Conclusiones: Las lesiones de glándulas salivales menores en nuestro hospital se caracterizan por ser predominantemente benignas. Se encontró similar frecuencia de tumores benignos y malignos. Predominó el adenoma pleomorfo entre los primeros y el carcinoma mucoepidermoide entre los segundos. Los tumores benignos, luego de su extirpación, se manejaron con cierre del lecho tumoral mediante rotación de colgajos locales de mucosa oral para cobertura y, en algunos casos, cierre por segunda intención. La evolución fue en general excelente. En el caso de los tumores malignos, el manejo dependió de la histología.

Palabras clave: Lesiones intraorales, glándulas salivales menores, adenoma pleomorfo, carcinoma mucoepidermoide.

\section{Introducción}

La patología de las glándulas salivales menores comprende lesiones como: anomalías del desarrollo, traumatismos, transtornos obstructivos, quistes, infecciones, enfermedades metabólicas, lesiones linfoepiteliales benignas y neoplasias ${ }^{1-4}$.

En cuanto a las neoplasias, éstas conforman un grupo heterogéneo de tumores con una gran variación histo-morfológica ${ }^{5-8}$.

La Organización Mundial de la Salud (OMS), clasifica a los tumores de glándulas salivales según su patrón histológico en: adenomas, carcinomas, lesiones pseudotumorales y otros tumores ${ }^{4-6,8-10}$.

La incidencia de los tumores de glándulas salivales es $4-8 \%$ de los tumores de cabeza y cuello.

Las neoplasias de glándulas salivales menores (GSMe) corresponden al 10-15\% del total de tumores de glándulas salivales. Se ha reportado igual frecuencia de tumores benignos y malignos.

El tumor benigno más encontrado es el adenoma pleomorfo y el maligno más frecuente es el carcinoma adenoide quístico ${ }^{5,8,11,12}$.

No existe claridad en cuanto a la etiopatogenia de estos tumores, pero se los ha relacionado con factores como: tabaco y exposición a radiación ionizante $\mathrm{i}^{13-16}$.

Desde el punto de vista histopatológico, se ha avanzado en el estudio de los tumores de GSMe, por medio de la inmunohistoquímica, genética molecular y el descubrimiento de nuevas neoplasias ${ }^{13,14,16,17}$.

Los factores pronósticos del cáncer de glándulas salivales en general están dados por: tipo histológico, tamaño tumoral, extensión local, linfonodos $(+)$, metástasis a distancia y recurrencia local y regional $^{9,18,19}$.

En cuanto al manejo del cáncer de GSM, la cirugía es el tratamiento a realizar, asociada a radioterapia, dependiendo de las características del tumor ${ }^{20,21}$.

El objetivo de la presente revisión es evaluar la incidencia, manejo y evolución de las lesiones intraorales de glándulas salivales menores tratadas por nuestro equipo.

\section{Material y Método}

En el presente estudio se analizó retrospectivamente el archivo de casos operados por lesiones intraorales de glándulas salivales menores, entre los años 1975 y 2009 en el Departamento de Cabeza y Cuello y Plástica Maxilo-Facial del Hospital Barros Luco-Trudeau, mediante la revisión de las fichas clínicas. Se estudiaron 59 pacientes, 48 con lesiones benignas (de las cuales 13 fueron neoplasias benignas) y 11 con neoplasias malignas.

El análisis de los datos se realizó con el programa FileMaker Pro 8.5.

Se presentan los resultados en porcentajes, promedios y desviaciones estándar.

\section{Resultados}

En nuestro estudio, se encontraron 59 pacientes con lesiones de glándulas salivales menores, 48 benignas y 11 malignas.

De las 48 lesiones benignas, 35 fueron lesiones no neoplásicas como: quistes salivales (28), sialoadenitis crónica (4) y linfoadenitis crónica inespecífica (3) y 13, neoplasias benignas. De las lesiones benignas no neoplásicas (35), la mayoría (28) fueron descritas como quistes salivales, los que se ubicaron preferentemente en el labio inferior y en segundo lugar en la mucosa yugal. El paladar fue la tercera localización más frecuente.

En cuanto a la sialoadenitis crónica, las lesiones se distribuyeron entre la mucosa yugal y vestibular al igual que la linfoadenitis crónica inespecífica.

El promedio de edad de los pacientes con lesiones benignas no neoplásicas fue de $33 \pm 1,7$ años (Tabla 1, Figuras 1 y 2).

En cuanto a las lesiones neoplásicas, del total de 24, 13 fueron benignas y 11 malignas. El promedio de edad para las lesiones neoplásicas benignas y malignas fue $49,7 \pm 21,3$ y 49,5 $\pm 23,8$ años respectivamente.

Dentro de las benignas, el tumor más encontrado 


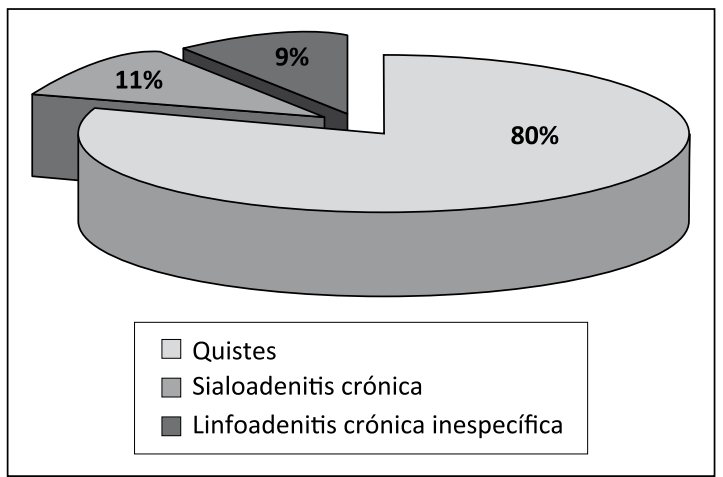

Figura 1. Distribución de las lesiones benignas no neoplásicas.

Tabla 1. Distribución de las lesiones benignas no neoplásicas

\begin{tabular}{|c|c|c|c|}
\hline Histología & $\mathbf{n}$ & Localización & $\begin{array}{c}\text { Edad } \\
\text { (promedio) }\end{array}$ \\
\hline $\begin{array}{l}\text { Quistes (de reten- } \\
\text { ción, mucoceles) }\end{array}$ & 28 & $\begin{array}{l}11 \text { labio inferior } \\
8 \text { mucosa yugal } \\
6 \text { paladar } \\
2 \text { labio superior } \\
1 \text { periamigdaliano }\end{array}$ & 31,4 \\
\hline $\begin{array}{l}\text { Sialoadenitis } \\
\text { crónica }\end{array}$ & 4 & $\begin{array}{l}2 \text { vestíbulo oral } \\
2 \text { mucosa yugal }\end{array}$ & 32,2 \\
\hline $\begin{array}{l}\text { Linfoadenitis } \\
\text { crónica inespecífica }\end{array}$ & 3 & $\begin{array}{l}2 \text { mucosa yugal } \\
1 \text { vestíbulo oral }\end{array}$ & 37,3 \\
\hline
\end{tabular}

fue el adenoma pleomorfo en un $62 \%$ de los pacientes (Tabla 2, Figuras 3 y 4 ).

Dentro de los cánceres, el más frecuente fue el carcinoma mucoepidermoide, en un $37 \%$ de los casos. La frecuencia del $\mathrm{Ca}$ adenoide quístico y el $\mathrm{Ca}$ de células acinosas fue de $18 \%$ cada uno.

Se encontró un caso de Ca espinocelular, adenocarcinoma y carcinoma indiferenciado respectivamente (Tabla 3, Figuras 5 y 6 ).

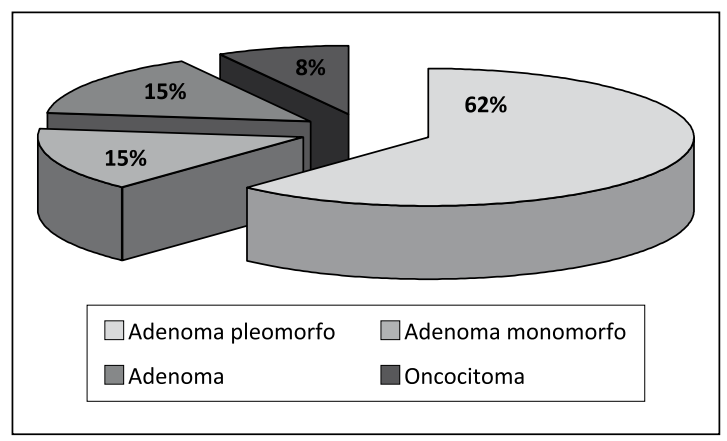

Figura 3. Diagnóstico histológico de las neoplasias benignas.

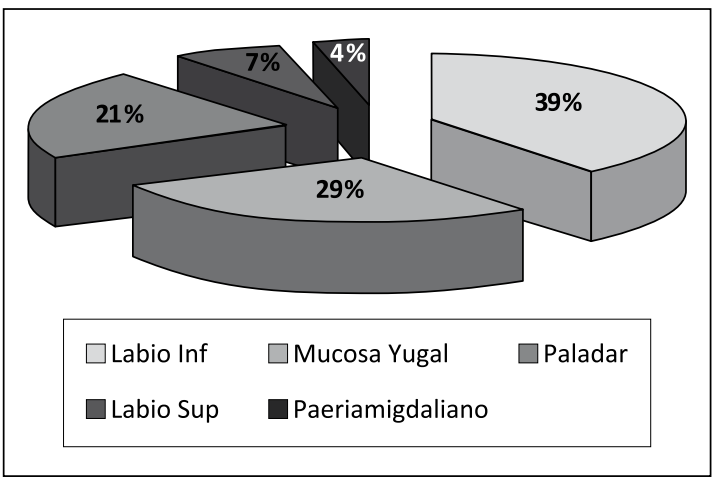

Figura 2. Localizaciones más frecuentes de las lesiones benignas no neoplásicas.

Tabla 2. Distribución y diagnóstico histológico de las neoplasias benignas

\begin{tabular}{|cccll|}
\hline & Sexo & Edad & Localización & Histología \\
1 & F & 59 & Velo palatino & Adenoma pleomorfo \\
2 & M & 28 & Paladar duro & Adenoma pleomorfo \\
3 & M & 42 & Labio superior & Adenoma pleomorfo \\
4 & F & 13 & Mucosa yugal & Adenoma pleomorfo \\
5 & M & 59 & Paladar & Adenoma pleomorfo \\
6 & F & 53 & Paladar duro y & Adenoma pleomorfo \\
7 & M & 69 & Paladar & Adenoma pleomorfo \\
& & & & metaplasia ep \\
8 & M & 64 & Paladar & Adenoma pleomorfo \\
9 & F & 26 & Mucosa yugal & Tumor monomórfico \\
& & & & mioepitelial \\
10 & F & 72 & Paladar blando & Adenoma pleomorfo \\
11 & M & 25 & Paladar duro & Adenoma monomórfico \\
12 & F & 83 & Paladar & Oncocitoma \\
13 & F & 54 & Paladar & Adenoma pleomorfo \\
\hline
\end{tabular}

$* \mathrm{M}=$ masculino, $\mathrm{F}=$ femenino.

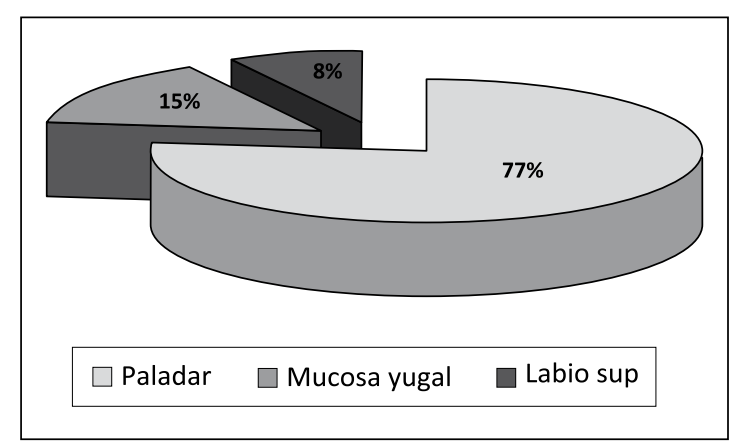

Figura 4. Localizaciones más frecuentes de las neoplasias benignas. 
M. A. MATAMALA S. y cols.

Tabla 3. Distribución y diagnóstico histológico del cáncer de GSMe

\begin{tabular}{|c|c|c|c|c|}
\hline & Sexo & Edad & Localización & Histología \\
\hline 1 & $\mathrm{~F}$ & 17 & Paladar duro & $\begin{array}{l}\text { CME malignidad } \\
\text { intermedia }\end{array}$ \\
\hline 2 & $\mathrm{M}$ & 21 & Paladar duro & $\begin{array}{l}\text { CME malignidad } \\
\text { intermedia }\end{array}$ \\
\hline 3 & M & 35 & Lengua & CME de bajo grado \\
\hline 4 & $\mathrm{~F}$ & 49 & Mucosa yugal & $\begin{array}{l}\text { CME malignidad } \\
\text { intermedia }\end{array}$ \\
\hline 5 & $\mathrm{~F}$ & 72 & Paladar & $\begin{array}{l}\text { Ca adenoide } \\
\text { quístico }\end{array}$ \\
\hline 6 & $\mathrm{~F}$ & 36 & Paladar & $\begin{array}{l}\text { Ca adenoide } \\
\text { quístico }\end{array}$ \\
\hline 7 & M & 87 & Mandíbula & $\begin{array}{l}\text { Ca de células } \\
\text { acinosas }\end{array}$ \\
\hline 8 & M & 29 & Paladar blando & $\begin{array}{l}\text { Ca de células } \\
\text { acinosas bajo grado }\end{array}$ \\
\hline 9 & M & 67 & Paladar & Adenocarcinoma \\
\hline 10 & $\mathrm{M}$ & 76 & $\begin{array}{l}\text { Pilar anterior } \\
\text { paladar }\end{array}$ & $\mathrm{Ca}$ espinocelular \\
\hline 11 & M & 56 & Paladar & $\begin{array}{l}\mathrm{Ca} \text { indif. en } \\
\text { adenoma pleomorfo }\end{array}$ \\
\hline
\end{tabular}

$* \mathrm{M}=$ masculino, $\mathrm{F}=$ femenino.

El carcinoma indiferenciado fue encontrado en un foco de adenoma pleomorfo.

En relación a la terapéutica para los cánceres de GSMe, los adenocarcinomas bien diferenciados (1) y los carcinomas mucoepidermoides de bajo grado (1) fueron los únicos manejados exclusivamente con cirugía, con confirmación histológica de márgenes libres. El resto de los tipos histológicos de carcinomas de GSMe (9) recibieron radioterapia post-operatoria (Figura 7).

Hubo 2 recidivas, uno de ellos en dos ocasiones; un $\mathrm{Ca}$ mucoepidermoide de malignidad intermedia infiltrante.

\section{Discusión}

De acuerdo con nuestro estudio, las lesiones intraorales de glándulas salivales menores son en su mayoría benignas, y dentro de éstas la mayoría son quísticas. Estas lesiones se presentaron en mayor proporción en menores de 40 años, lo que coincide con lo reportado en la literatura ${ }^{1-4}$.

En cuanto a las neoplasias de glándulas salivales menores, éstas son raras y su verdadera frecuencia e incidencia no están claras. Por este motivo, la ma-

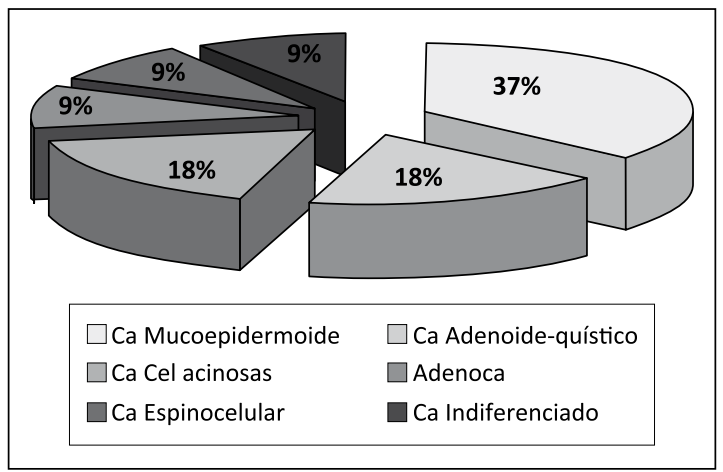

Figura 5. Diagnóstico histológico de los cánceres de GSMe.

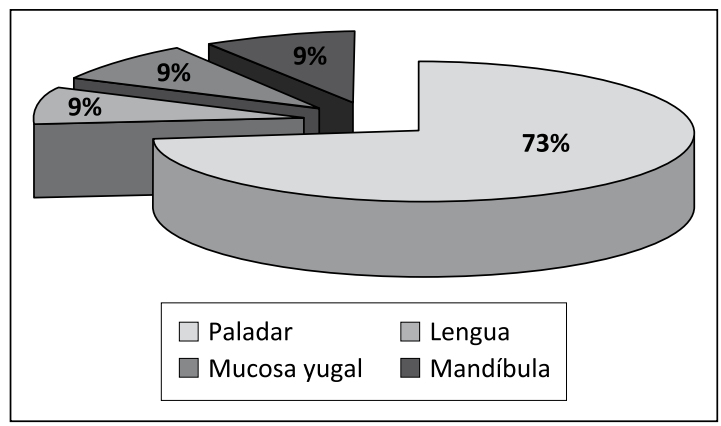

Figura 6. Localizaciones más frecuentes de los cánceres de GSMe.

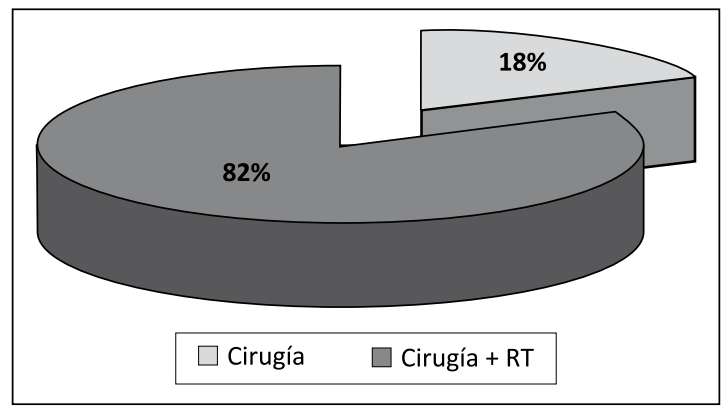

Figura 7. Manejo de los cánceres de GSMe.

yoría de los estudios reportados en la literatura son retrospectivos con series pequeñas ${ }^{5-7,9,22}$.

En nuestra serie se encontró una frecuencia similar entre tumores benignos y malignos, a diferencia de la mayoría de los reportes, en que se describe a los tumores benignos como los más frecuentes s,23-26. $^{8}$.

$\mathrm{Al}$ igual que en la mayor parte de los estudios analizados, el tumor benigno más frecuente encontrado en nuestra serie fue el adenoma pleomorfo. El sitio más frecuente de localización para los tumores benignos fue el paladar, lo que coincide con lo encontrado en las distintas series ${ }^{8,23}$. 
No existe consenso en cuanto a la neoplasia maligna más frecuente, los reportes se dividen entre el carcinoma mucoepidermoide y el adenoideo quístico. En nuestro trabajo, el carcinoma mucoepidermoide fue el más frecuente.

Los carcinomas adenoideo quístico y el de células acinosas se encontraron en igual proporción.

El paladar fue el sitio más frecuente de localización, lo que concuerda con la mayoría de los reportes ${ }^{8,23}$.

En cuanto al diagnóstico preoperatorio, se ha descrito la punción con aguja fina (PAAF) para estas lesiones, con una relativa baja sensibilidad, con rangos entre 29 y $67 \%$ y una alta especificidad, que va de un 84 a 100\%. La PAAF estaría justificada en casos que se trata de descartar una lesión de manejo médico como un linfoma o en pacientes con contraindicación quirúrgica ${ }^{13,27}$. En nuestra revisión, no se describe diagnóstico citológico preoperatorio.

Según estudios histopatológicos, la transformación maligna de los tumores de glándulas salivales puede darse a partir de un tumor benigno, progresar desde un carcinoma de bajo grado, por des-diferenciación y por invasión estromal de un carcinoma in $s i t u^{28,29}$. Encontramos un caso de carcinoma indiferenciado en un adenoma pleomorfo.

Los distintos reportes coinciden en que el manejo de los tumores de glándulas salivales está dado por la cirugía con márgenes adecuados, y en el caso de los malignos, asociado a radioterapia según las características del tumor ${ }^{10,21,30}$.

En nuestro centro, los tumores benignos, luego de su extirpación se manejaron con cierre del lecho tumoral mediante rotación de colgajos locales de mucosa oral para cobertura y, en algunos casos, cierre por segunda intención. La evolución fue, en general satisfactoria, con seguimiento de 3 a 14 meses.

En el caso de los tumores malignos, el manejo dependió de las características histológicas.

Tumores como adenocarcinomas bien diferenciados y carcinomas mucoepidermoides de bajo grado de malignidad, se trataron sólo con cirugía. El resto de los tumores malignos recibieron radioterapia postoperatoria. Hubo 2 recidivas, uno de ellas en dos ocasiones.

En base a los hallazgos de este trabajo, podemos deducir que:

- Las lesiones intraorales de GSMe tratadas en el HBLT, son en su mayoría benignas.

- La lesión intraoral benigna no neoplásica de GSMe más frecuente en nuestra serie fue el mucocele.

- El adenoma pleomorfo fue la neoplasia benigna más frecuente y el carcinoma mucoepidermoide el cáncer más encontrado.
- Las lesiones neoplásicas benignas y malignas se encuentran en igual proporción.

- Se debe mantener un seguimiento estricto de los pacientes post operados por adenoma pleomorfo, dado el conocimiento actual sobre posibilidad de malignización y la alta recidiva.

\section{Referencias}

1. Zhao YF, Jia J, Jia Y. Complications Associated With Surgical Management of Ranulas. J Oral Maxillofac Surg 2005;63:51-4.

2. Baurmash HD. Submandibular Salivary Stones: $\mathrm{Cu}-$ rrent Management Modalities. J Oral Maxillofac Surg. 2004;62:369-78.

3. Alcure ML, Della Coletta R, Graner E, Di Hipolito O Jr, Lopes MA. Sialolithiasis of minor salivary glands: a clinical and histopathological study. Gen Dent. 2005;53:278-81.

4. Ono Y, Takahashi H, Inagi K, Nakayama M, Okamoto M. Clinical study of benign lesions in the oral cavity. Acta Otolaryngol Suppl. 2002;547:79-84.

5. Yih WY, Kratochvil FJ, Stewart JC. Intraoral minor salivary gland neoplasms: review of 213 cases. J Oral Maxillofac Surg. 2005;63:805-10.

6. Jaber MA. Intraoral minor salivary gland tumors: a review of 75 cases in a Libyan population. Int J Oral Maxillofac Surg. 2006;35:150-4.

7. Buchner A, Merrell PW, Carpenter WM. Relative frequency of intra-oral minor salivary gland tumors: a study of 380 cases from northern California and comparison to reports from other parts of the world. J Oral Pathol Med. 2007;36:207-14.

8. Pires FR, Pringle GA, De Almeida OP, Chen SY. Intraoral minor salivary gland tumors: a clinicopathological study of 546 cases. Oral Oncol. 2007;43:463-70.

9. Copelli C, Bianchi B, Ferrari S, Ferri A, Sesenna E. Malignant tumors of intraoral minor salivary glands. Oral Oncol. 2008;44:658-63.

10. Ávila RE, Samar ME, Camps D, Fernández R, Fernández JE. Tumores de glándulas salivales menores. Estudio retrospectivo de 46 casos. Rev Esp Patol. 2008;41:23-9.

11. Olsen KD, Lewis JE. Carcinoma ex pleomorphic adenoma: a clinicopathologic review. Head Neck 2001;23:705-12.

12. Mücke T, Robitzky LK, Kesting MR, Wagenpfeil S, Holhweg-Majert B, Wolff KD, et al. Advanced malignant minor salivary glands tumors of the oral cavity. Oral Surg Oral Med Oral Pathol Oral Radiol Endod. 2009;108:81-9.

13. Greer RO. Pathology of malignant and premalignant oral epithelial lesions. Otolaryngol Clin North Am. 2006;39:249-75.

14. Andreadis D, Epivatianos A, Poulopoulos A, Nomikos 
M. A. MATAMALA S. y cols.

A, Papazoglous G, Antoniades D, et al. Detection of C-KIT (CD117) molecule in benign and malignant salivary gland tumours. Oral Oncol. 2006;42:57-65.

15. Uro-Coste E. 2009 update in salivary gland tumoral pathology. Ann Pathol. 2009;29:274-85.

16. Monteiro LS, Palmeira C, Bento MJ, Lopes C. DNA content in malignant salivary gland tumours. Oral Dis. 2009; 15:295-301.

17. Shi L, Chen XM, Wang L, Zhang L, Chen Z. Expression of Caveolin-1 in Mucoepidermoid Carcinoma of the Salivary Glands: Correlation With Vascular Endothelial Growth Factor, Microvessel Density, and Clinical Outcome. Cancer 2007;109:1523-31.

18. Moreno M, Moreno A, Tomé C. Sentinel lymph node biopsy in adenocarcinoma of minor salivary gland. Oral Oncol. 2008;44:305-8.

19. Loh KS, Barker E, Bruch G, O’Sullivan B, Brown DH, Goldstein DP, et al. Prognostic factors in malignancy of the minor salivary glands. Head Neck 2009;31:58-63.

20. Bell RB, Dierks EJ, Homer L, Potter BE. Management and outcome of patients with malignant salivary gland tumors. J Oral Maxillofac Surg. 2005;63:917-28.

21. Le QT, Birdwell S, Terris DJ, Gabalski EC, Varghese A, Fee W Jr, et al. Postoperative irradiation of minor salivary gland malignancies of the head and neck. Radiother Oncol. 1999;52:165-71.

22. Ladeinde AL, Adeyemo WL, Ogunlewe MO, Ajayi OF, Omitola OG. Salivary gland tumours: a 15 -year review at the Dental Centre Lagos University Teaching Hospi- tal. Afr J Med Med Sci. 2007;36:299-304.

23. Wang D, Li Y, He H, Liu L, Wu L, He Z. Intraoral minor salivary gland tumors in a Chinese population: a retrospective study on 737 cases. Oral Surg Oral Med Oral Pathol Oral Radiol Endod. 2007;104:94-100.

24. Gbotolorun OM, Arotiba GT, Effiom OA, Omitola OG. Minor salivary gland tumours in a Nigerian hospital: a retrospective review of 146 cases. Odontostomatol Trop. 2008;31:17-23.

25. Targa-Stramandinoli R, Torres-Pereira C, Piazzetta CM, Giovanini AF, Amenábar JM. Neoplasias de glándulas salivales menores: estudio de 10 años. Acta Otorrinolaringol Esp. 2009;60:199-201.

26. Pons-Vicente O, Almendros-Marqués N, Berini-Aytés L, Gay-Escoda C. Minor salivary gland tumors: A clinicopathological study of 18 cases. Med Oral P Patol Oral Cir Bucal. 2008;13:E582-8.

27. Cohen EG, Patel SG, Lin O, Boyle JO, Kraus DH, Singh B, et al. Fine-Needle Aspiration Biopsy of Salivary Gland lesions in a Selected Patient Population. Arch Otolaryngol Head Neck Surg. 2004;130:773-8.

28. Krahl D, Altenburg A, Zouboulis CC. Reactive hyperplasias, precancerous and malignant lesions of the oral mucosa. J Dtsch Dermatol Ges. 2008;6:217-32.

29. Cheuk W, Chan JK. Advances in salivary gland pathology. Histopathology 2007;51:1-20.

30. Rinaldo A, Shaha AR, Pellitteri PK, Bradley PJ, Ferlito A. Management of malignant sublingual salivary gland tumors. Oral Oncology 2004;40:2-5. 